\title{
Theoretical Design and Analysis of EDFA Gain Control System Based on Two-Level EDFA Model
}

\author{
Seong-Ho SONG, Seop-Hyeong PARK \\ Hallym University, \\ 1 Okchon, Chunchon, Gangwon 200-701, Korea, \\ ssh@hallym.ac.kr, spark@hallym.ac.kr
}

\begin{abstract}
In this paper, a disturbance observer (DOB) based erbium-doped fiber amplifier(EDFA) gain controller is proposed. In order to stabilize an EDFA gain in wavelength-division-multiplexing (WDM) channel add/drop networks, the channel add/drop is estimated based on a mathematical EDFA model and compensated using DOB technique. The performance of the proposed control method is theoretically analyzed using Lyapunov method. The performance of the proposed DOB-based gain control algorithm was investigated through simulations. Simulation results verify the excellent performance of the proposed gain control methodology.
\end{abstract}

Keywords: Erbium-Doped Fibre Amplifier, Gain Control, Mathematical Model, Disturbance Observer, Channel Add/Drop. Theoretical Analysis

\section{Introduction}

Control and management of erbium-doped fiber amplifiers (EDFAs) is an important design problem in the evolution towards a dynamic optical network [1]. As wavelength- division multiplexed networks increase in complexity, there remain a number of challenges, such as dealing with disturbances related to intrinsic network characteristics. The power level of each channel in WDM networks should be unchanged when channel add/drops or active rearrangements of network occur. Keeping the signal powers to a constant value is more important when the signals are amplified through EDFAs. At the EDFA, the change of the number of signals causes the change of the amplifier gain of each signal due to the cross gain saturation effect $[2,3,4]$ which results in gain-related errors at the receivers.

To avoid this effect, several control methods have been developed [5-9]. One of them uses EDFA output as a feedback signal in an optical feedback control loop [5]. The all-optical scheme has a drawback; the frequency of channel add/drop should be less than that of the relaxation oscillation frequency of EDFA, which is several hundred $\mathrm{Hz}$. On the other hand, the mostly used one is an electrical scheme which controls the pump laser output electrically according to EDFA output signal level $[9,10]$.

Based on $H_{\infty}$ control theory, a systematic design approach has been introduced in [9] and some robustness has been also analyzed therein. However, most systems adopt traditional proportional, integral, derivative
(PID) control, or need feed-forward perfect cancellation algorithms for gain control. As mentioned in [9], there have been no formal attempts to analyze the performance theoretically and systematically.

In this paper, theoretical performance analysis of EDFA gain control algorithm is carried out based on a mathematical EDFA model. As a nominal controller, a proportional-,integral controller is considered and a disturbance observer is considered as feed-forward control. To our knowledge, this is the first attempt to theoretically analyze the performance of PI and feed forward control algorithm in EDFA control literature.

Singular perturbation approach [11] reduces the mathematical model to two level equation and disturbance observer technique[12,13] is adopted to compensate channel add/drop effects in this two-level model. The asymptotic performance is proven theoretically using Lyapunov method and through simulation results, the feasibility of the proposed algorithm is verified.

\section{Mathematical Model of EDFA}

In order to design an EDFA gain controller, the following three-level model is considered [14]. The energy level of EDFA is shown in Fig.1 and the equations for three-level process are derived as follows.

$$
\begin{aligned}
& \frac{d N_{3}}{d t}=-\Gamma_{32} N_{3}-\left(N_{3} \sigma_{p}^{e}-N_{1} \sigma_{p}^{a}\right) \phi_{p} \\
& \frac{d N_{2}}{d t}=-\Gamma_{21} N_{2}+\left(N_{1} \sigma_{s}^{a}-N_{2} \sigma_{s}^{e}\right) \phi_{S}+\Gamma_{32} N_{3}
\end{aligned}
$$


$\frac{d N_{1}}{d t}=\Gamma_{21} N_{1}-\left(N_{1} \sigma_{s}^{a}-N_{2} \sigma_{s}^{e}\right) \phi_{s}+\left(N_{3} \sigma_{p}^{e}-N_{1} \sigma_{p}^{a}\right) \phi_{p}$

where $\phi_{\mathrm{s}}, \phi_{\mathrm{p}}$ are photon flux densities per second of a signal and a pump, $\sigma_{s}^{e}, \sigma_{s,}^{a} \sigma_{p}^{e}, \sigma_{p}^{a}$ are absorption and emission cross section of a signal and a pump $\left(\sigma^{\mathrm{T}}=\sigma^{\mathrm{e}}+\sigma^{\mathrm{a}}\right) . \mathrm{N}_{1}, \mathrm{~N}_{2}$, and $\mathrm{N}_{3}$ are the number of erbium-ions at each energy level $\left(N=N_{1}+N_{2}+N_{3}=1\right)$.

$$
\begin{aligned}
& \frac{d P_{p}}{d t}=\rho \Gamma_{p}\left(\sigma_{p}^{T} N_{3}+\sigma_{p}^{a} N_{2}-\sigma_{p}^{a}\right) P_{p} \\
& \frac{d P_{S}}{d t}=\rho \Gamma_{s}\left(\sigma_{s}^{T} N_{2}+\sigma_{s}^{a} N_{3}-\sigma_{s}^{a}\right) P_{S}
\end{aligned}
$$

$\mathrm{P}_{\mathrm{s}}$ and $\mathrm{P}_{\mathrm{p}}$ are respectively the power of the signal and the pump, $\rho$ is an erbium density, and $\Gamma \mathrm{s}$ and $\Gamma \mathrm{p}$ are respectively the geometric correction factor for the overlap between the power and the erbium-ions.

Define a reservoir $r(t)$ that represents the number of excited erbium-ions at each level and the EDFA gain of $k$-th channel as follows.

$$
\begin{aligned}
& r_{i}(t) \equiv \rho A \int_{0}^{L} N_{i}(z, t) d z, i=2,3 \\
& G_{k}(t)=\ln \frac{P_{k}^{\text {out }}}{P_{k}^{\text {in }}}
\end{aligned}
$$

where $L$ is the length of the erbium-doped fiber and $A$ is the cross-section area of erbium-doped fiber core. By integrating (6) along the whole length of EDF, we can obtain the following equation. From the definitions of reservoir $r(t)$ and $G_{k}(t)=\ln \left(P_{k}{ }^{\text {out }} / P_{k}{ }^{i n}\right)$, equations (1)-(5) are changed into the following equations.

$$
\begin{aligned}
& \frac{d r_{3}}{d t}=-\Gamma_{32} r_{3}+P_{p}^{i n}(t) \\
& \frac{d r_{2}}{d t}=-\Gamma_{21} r_{2}+\Gamma_{32} r_{3}+\sum_{k=1}^{N}\left(1-e^{G_{k}(t)}\right) P_{k}^{i n}(t) \\
& G_{k}(t)=B_{k} r_{2}-A_{k}
\end{aligned}
$$

where

$$
\phi_{p}=\Gamma_{p} \frac{P_{p}}{A}, \phi_{S}=\Gamma_{s} \frac{P_{p}}{A}, A_{k}=\rho \Gamma_{k} \sigma_{k}^{a} L, B_{k}=\frac{\Gamma_{k} \sigma_{k}^{T}}{A}
$$

From (8) and (9), three-level EDFA model equations are obtained as follows.

$$
\begin{aligned}
& \frac{\dot{G}_{k}}{B_{k}}=-\Gamma_{21} \frac{G_{k}}{B_{k}}+\Gamma_{32} r_{3}+\sum_{k=1}^{N}\left(1-e^{G_{k}(t)}\right) P_{k}^{i n}(t)-\Gamma_{21} \frac{A_{k}}{B_{k}} \\
& \dot{r}_{3}=-\Gamma_{32} r_{3}+P_{p}^{i n}
\end{aligned}
$$

Generally, $\Gamma_{32}$ is very large compared with $\Gamma_{21}$, so three-level model can be reduced to the following two-level model equation by singular perturbation method[10].

$$
\begin{aligned}
& \frac{\dot{G}_{k}}{B_{k}}=-\Gamma_{21} \frac{G_{k}}{B_{k}}+P_{p}^{i n}(t)+\sum_{k=1}^{N}\left(1-e^{G_{k}(t)}\right) P_{k}^{i n}(t)-\Gamma_{21} \frac{A_{k}}{B_{k}}(14) \\
& 0=-\Gamma_{32} r_{3}+P_{p}^{i n}
\end{aligned}
$$

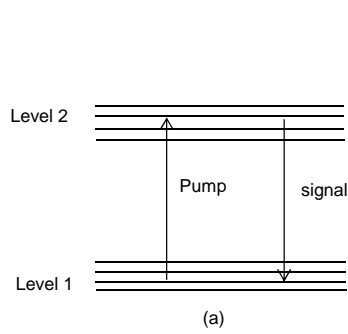

(a) two-level model

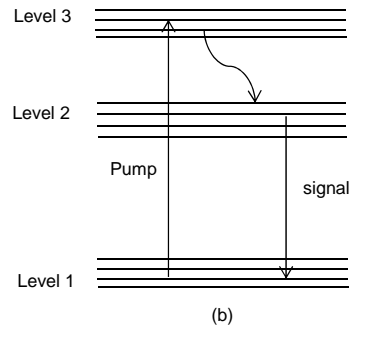

(b) three level model
Figure 1. Models of EDFA:

\section{Design and Analysis of EDFA Gain Control}

\subsection{Nominal controller design}

Two-level model (14) can be rewritten as the followings.

$\frac{d}{d t} G_{k}=-\Gamma_{21} G_{k}+B_{k} P_{p}^{i n}(t)+B_{k} d(t)$

where we define a $d(t)$ as follows, which is considered as a disturbance.

$$
d(t)=\sum_{k=1}^{N}\left(1-e^{G_{k}(t)}\right) P_{k}^{i n}(t)-\Gamma_{21} \frac{A_{k}}{B_{k}}
$$

In general, the variation of $d(t)$ means channel add/drops. The transfer function from a pump input to a channel gain, $\mathrm{P}(\mathrm{s})$ represents the nominal EDFA plant model with no disturbance.

$$
P(s)=\frac{L\left[\hat{G}_{k}\right]}{L\left[P_{p}^{i n}\right]}=\frac{B_{k}}{s+\Gamma_{21}}
$$

where L[.] is a laplace transform. Since the nominal plant (18) is a first-order linear system, we adopt a nominal controller with a PI control as follows in order to stabilize EDFA channel gains.

$$
C(s)=K_{p}+\frac{K_{I}}{s}
$$


PI controller can achieve the set-point tracking and arbitrary pole-placement for the system (18). So, if channel add/drops do not exist, the performance of closed-loop control system can be controlled as much as we want.

\subsection{Compensation of channel add/drop effect: disturbance observer technique}

In order to cope with channel add/drop effect, the variation of $d(t)$ defined by (17) should be estimated and compensated. Disturbance observer technique can be applied to the estimation and compensation of $\mathrm{d}(\mathrm{t})$.

From (19), define a control input

$$
P_{p}^{i n}(t)=K_{p}\left(G_{k}^{c}-G_{k}\right)+K_{I} \int_{0}^{t}\left(G_{k}^{c}-G_{k}\right) d t-\hat{d}(t)(20)
$$

Figure 2 shows the closed-loop system of EDFA control system with PI control and DOB.

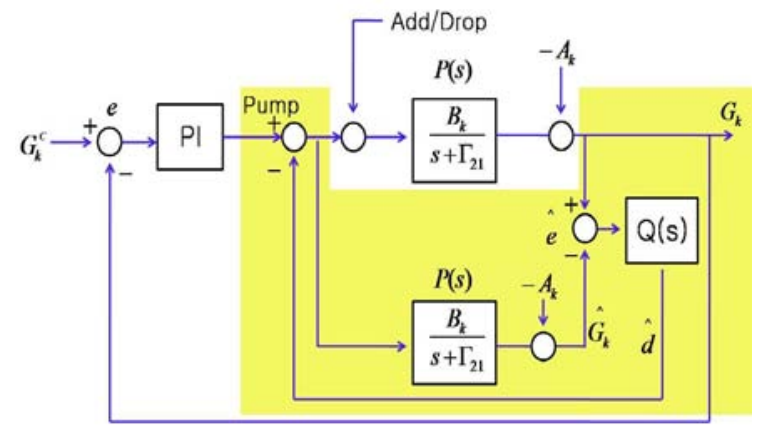

Figure 2. Closed-loop control system

As you can see, $\hat{G}_{k}$ is a channel gain estimate when no $\mathrm{d}(\mathrm{t})$ exist and $G_{k}-\hat{G}_{k}$ contains the information of a disturbance $\mathrm{d}(\mathrm{t})$. Thus, the estimate $\hat{d}$ can be achieved by properly filtering this. This filter $\mathrm{Q}(\mathrm{s})$ is designed to make $\hat{d}$ to be a loss-pass filtered signal of $\mathrm{d}(\mathrm{t})$.

$$
\frac{\hat{d}(s)}{d(s)}=P(s) Q(s)=\frac{\bar{A}}{s+\bar{A}}
$$

where $\hat{d}(s)$ and $d(s)$ are respectively Laplace transform of $\hat{d}(t)$ and $d(t)$. So, the filter $\mathrm{Q}(\mathrm{s})$ should be designed so that it satisfies the following equation.

$$
Q(s)=\frac{\bar{A}\left(s+\Gamma_{21}\right)}{B_{k}(s+\bar{A})}
$$

Using (21) and (22), a disturbance observer is implemented to estimate $\hat{d}(t)$ which compensate d(t) in (16).

\subsection{Stability analysis}

From (16), (20), and (21), the closed-loop system equations are described as follows.

$\frac{d}{d t} G_{k}=-\Gamma_{21} G_{k}+B_{k} P_{p}^{i n}(t)+B_{k} d(t)$

$\frac{d}{d t} \hat{d}(t)=-\bar{A}(\hat{d}-d)$

If we choose the observer time constant $\bar{A}$ as large as we can and apply singular perturbation method,

$0=\hat{d}-d$

Then, we have the following reduced dynamic equation of EDFA.

$\frac{d}{d t} \bar{G}_{k}=-\Gamma_{21} \bar{G}_{k}+B_{k} \bar{P}_{p}^{i n}(t)+B_{k} \bar{d}(t)$

where

$$
\begin{aligned}
& \bar{d}(t)=\hat{\bar{d}}=\sum_{k=1}^{N}\left(1-e^{\bar{G}_{k}(t)}\right) P_{k}^{i n}-\Gamma_{21} \frac{\left(A_{k}+G_{k}^{C}\right)}{B_{k}} \\
& \bar{P}_{p}^{i n}(t)=K_{p}\left(G_{k}^{c}-\bar{G}_{k}\right)+K_{I} \int_{0}^{t}\left(G_{k}^{c}-\bar{G}_{k}\right) d t-\hat{\bar{d}}(t)
\end{aligned}
$$

From (27) and (28), (26) can be rewritten as follows.

$$
\frac{d}{d t} \bar{G}_{k}=-\left(\Gamma_{21}+B_{k} K_{p}\right)\left(\bar{G}_{k}-G_{k}^{C}\right)-B K_{I} \int_{0}^{t}\left(\bar{G}_{k}-G_{k}^{C}\right) d t
$$

The input-output response given by (28) is described by the following typical second-order linear system response which is stable.

$\mathrm{L}\left[\bar{G}_{k}(t)\right]=\frac{B K_{I}}{s^{2}+\left(\Gamma_{21}+B_{k} K_{p}\right) s+B K_{I}} \mathrm{~L}\left[G_{k}^{C}\right]$

Suppose that the following assumption is satisfied.

(A1) Control gains $K_{p}$ and $K_{I}$ are chosen such that the following characteristic equation has roots with negative real parts.

$$
s^{2}+\left(\Gamma_{21}+B_{k} K_{p}\right) s+B K_{I}=0
$$

The assumption (A1) and (28) imply that there exist positive constants $\gamma_{G}, \gamma_{I}$ and $\sigma$ satisfying the following inequality. 


$$
\begin{aligned}
& \left|G_{k}^{c}-\bar{G}_{k}(t)\right| \leq \gamma_{G}\|\Theta(0)\| e^{-\sigma t} \\
& \left|\int_{0}^{t}\left(G_{k}^{c}-\bar{G}_{k}(t)\right) d t\right| \leq \gamma_{I}\|\Theta(0)\| e^{-\sigma t}
\end{aligned}
$$

where

$$
\Theta(t)=\left[G_{k}^{c}-\bar{G}_{k}(t) \int_{0}^{t}\left(G_{k}^{c}-\bar{G}_{k}(t)\right) d t\right]^{T}
$$

In order to analyze the performance of the proposed controller, we define error dynamics. Define error variables as follows.

$$
e_{1}=\int_{0}^{t}\left(G_{k}-\bar{G}_{k}\right) d t, e_{2}=G_{k}-\bar{G}_{k}, e_{3}=\hat{d}-d
$$

Then, we have the following error equations using (16), (17), (20) and (28).

$$
\begin{aligned}
\frac{d}{d t} e_{1} & =e_{2} \\
\frac{d}{d t} e_{2} & =-\left(\Gamma_{21}+B_{k} K_{p}\right) e_{2}-B_{k} K_{I} e_{1}-B_{k} e_{3} \\
\frac{d}{d t} e_{3} & =-(A-K(t)) e_{3}-K(t)\left\{-\frac{\Gamma_{21}+B_{k} K_{p}}{B_{k}}\left(e_{2}+\bar{G}_{k}-G_{k}^{C}\right)\right\} \\
& +K(t) K_{I 1}\left(e_{1}+\int_{0}^{t}\left(\bar{G}_{k}-G_{k}^{C}\right) d t\right)
\end{aligned}
$$

where

$$
K(t)=\sum_{n=1}^{N} B_{n} e^{G_{n}(t)} P_{n}^{i n}
$$

Since the length of EDF is finite, the reservoir $r_{2}$ is bounded and also $G_{n}(t)$ is bounded. So, there is a positive constant $\bar{K}$ such that

$$
K(t) \leq \bar{K}, \forall t \geq 0 .
$$

The assumption (A1) implies that there exist a positive definite matrix $P_{1}$ and $Q_{1}$ satisfying the following Lyapunov equation.

$$
A_{1}^{T} P_{1}+P_{1} A_{1}=-Q_{1}
$$

where

$$
A_{1}=\left[\begin{array}{cc}
0 & 1 \\
-B K_{1} & -\left(\Gamma_{21}+B_{k} K_{p}\right)
\end{array}\right]
$$

Now, we have the following theorem for the stability of the proposed control system.

\section{Theorem 1.}

Assume that the following matrix inequality holds for design parameters $K_{p}, K_{I}$ and $A$.

$$
\left[\begin{array}{cc}
-\lambda_{m}(Q) & \left.\left|P_{1} B\right|+\bar{K} \sqrt{K_{I}^{2}+\left(\frac{\Gamma_{21}}{B_{k}}+K_{p}\right)}\right) \\
\left\|P_{1} B\right\| \bar{K} \sqrt{K_{I}^{2}+\left(\frac{\Gamma_{21}}{B_{k}}+K_{p}\right)} & -\bar{A}+\bar{K}
\end{array}\right]<0
$$

Then, the error dynamics described in (34) is asymptotically stable.

The proof of Theorem 1 is given in Appendix. From Theorem 1, the error variable $e_{2}=G_{k}-\bar{G}_{k}$ goes to 0 and this with (32) finally guarantees that the EDFA gain $G_{k}$ is asymptotically converges to the desired gain $G_{k}^{C}$.

In order to design a gain controller, we first choose PI controller gains $K_{p}$ and $K_{I}$ such that $A_{1}$ is stable. Then, choose DOB gain $\bar{A}$ satisfying (38) or (39).

$$
-\bar{A}+\bar{K}+\frac{1}{\lambda_{m}(Q)}\left(\left\|P_{1} B\right\|+\bar{K} \sqrt{K_{I}^{2}+\left(\frac{\Gamma_{21}}{B_{k}}+K_{p}\right)}\right)^{2}<0
$$

\section{Simulation Results}

In simulations, the wavelength of the pump Laser is $980 \mathrm{~nm}$ and its maximum output power is $200 \mathrm{~mW}$. As signals, two channel signals with 1550nm and 1560nm wavelengths are applied to the system. Through simulations, we compare the performance of the proposed DOB based control algorithm to conventional PI control algorithm.

In the simulations, the desired channel 1 signal gain is set to $6 \mathrm{~dB}$. In order to compare the responses, we consider two cases depending on the PI control parameter values. The control parameters $K_{p}$ and $K_{I}$ are chosen such that the following equation holds for desired damping ratios $\zeta$ and natural frequencies $\omega_{n}$.

$$
s^{2}+\left(\Gamma_{21}+B_{k} K_{p}\right) s+B K_{I}=s^{2}+2 \zeta \omega_{n} s+\omega_{n}^{2}
$$

In both cases, damping ratios $\zeta$ is chosen to be 0.7 which causes a small overshoot to speed up the response a little bit. In order to compare the responses, we consider two natural frequencies $\omega_{n}=100000 \quad(\mathrm{rad} / \mathrm{sec})$ and $\omega_{n}=300000$ (rad/sec). The disturbance observer gain $\bar{A}$ is also chosen to be 4000000 for both cases which 
is at least ten times larger than the natural frequency of desired system.

For the performance analysis, $3.5 \mathrm{~mW}$ channel 2 signal drop occurs at 200usec and $2 \mathrm{~mW}$ channel 2 signal add takes place at 600usec.

Figure 3 shows the gain response results when $\omega_{n}=100000$ (rad/sec). As shown in the graph, figure (a) says that the gain response of the proposed algorithm (solid line) is stabilized faster than conventional PI controller without DOB(dotted line). Figure (b) shows the transient response and it is known that the characteristics of the response (solid line) converge to the desired second order system response (dotted line). From the figure, the settling times are 100usec in case of PI control without DOB and about 50usec in case of the proposed algorithm.

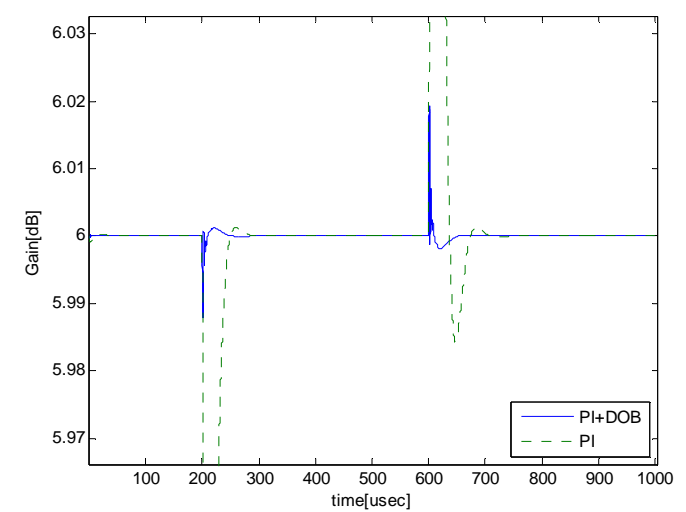

(a) Gain Response due to channel signal add/drop

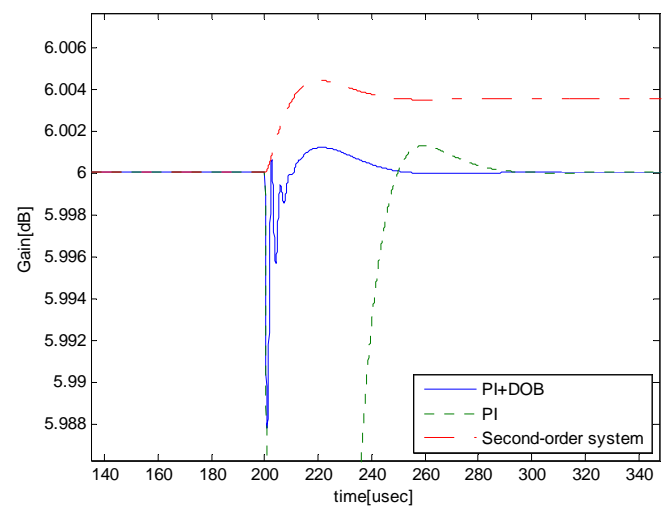

(b) Transient response of channel 1

Figure 3. Gain Response of Channel 1 in case of channel 2 signal add/drop $\left(\omega_{n}=100000\right)$

Figure 4 and Figure 5 shows the response of control algorithms when $\omega_{n}=300000$ (rad/sec). From (a) of Fig.4, the performance of the proposed DOB based PI control algorithm (solid line) is much better than conventional PI control algorithm without DOB (dotted line). The settling time of channel 1 signal gain for the PI control without DOB is about 50 usec. On the other hand, the settling time of channel 1 signal gain for the proposed control algorithm is less than 10usec which is 5 times faster. When DOB is adopted, the gain fluctuation due to signal adds is also improved 90\% rather than PI control. As theoretically analyzed in section 3.3, figure (b) of Figure4 shows that the gain response of the proposed algorithm (solid line) rapidly converges to the response of desired second order system (dash-dotted line) derived as a reduced system after transient response decays. Figure (c) of Figure 4 is the result of channel add/drop signal estimation using DOB. Since DOB has been designed based on simplified two-level EDFA model, the estimation result showed some oscillations even though the overall DOB input-output characteristics from channel add/drop input to its estimation output are represented by a first-order linear system. It is because of the controller design based on simplified model and this ignored dynamics would cause oscillations.

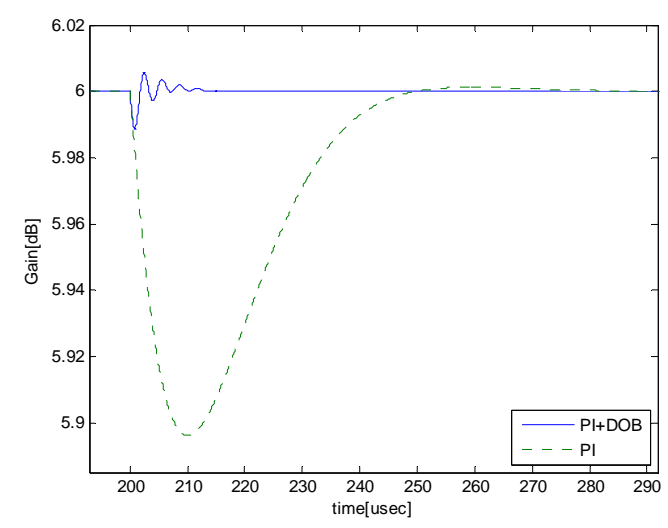

(a) Gain Response

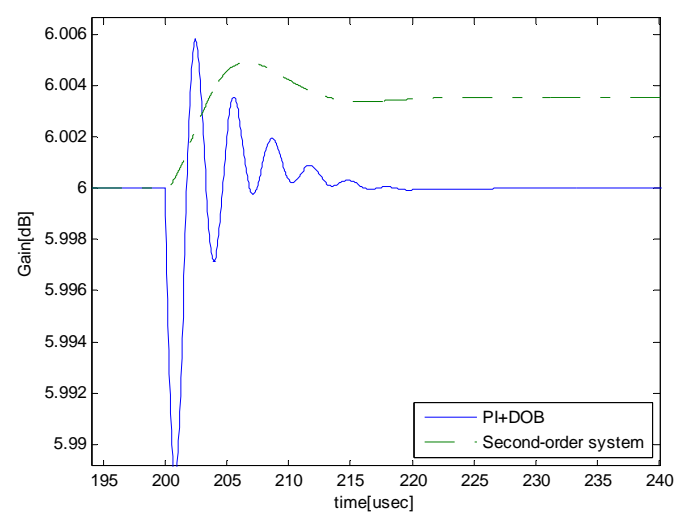

(b) Transient Response 


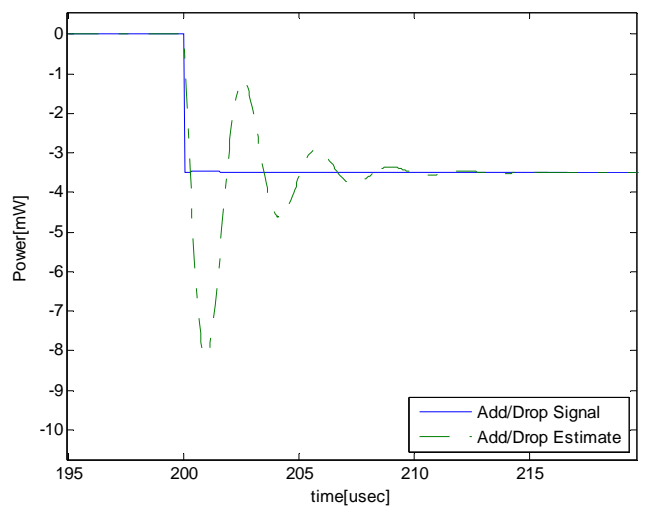

(c) Add/Drop Signal Estimation

Figure 4. Gain Response of Channel 1 in case of $3.5 \mathrm{~mW}$ channel 2 signal addition $\left(\omega_{n}=300000\right)$

Figure 5 shows the response when $3.5 \mathrm{~mW}$ channel 2 signal is dropped. The response characteristics are the same as in Figure 4 that the settling time is less than 10usec for the proposed algorithm.

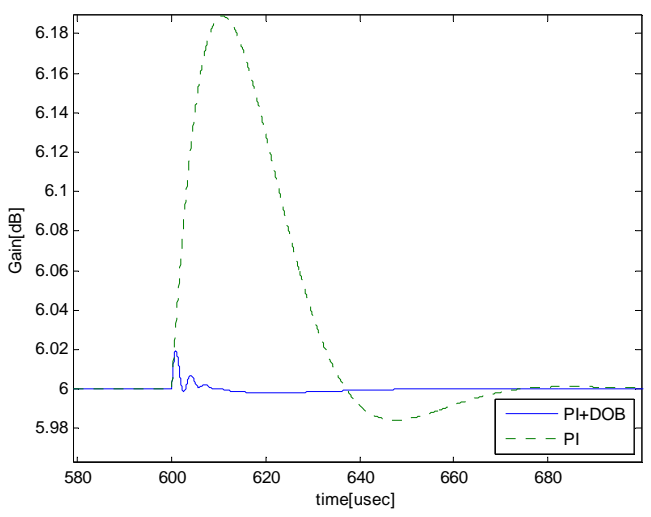

(a) Gain Response

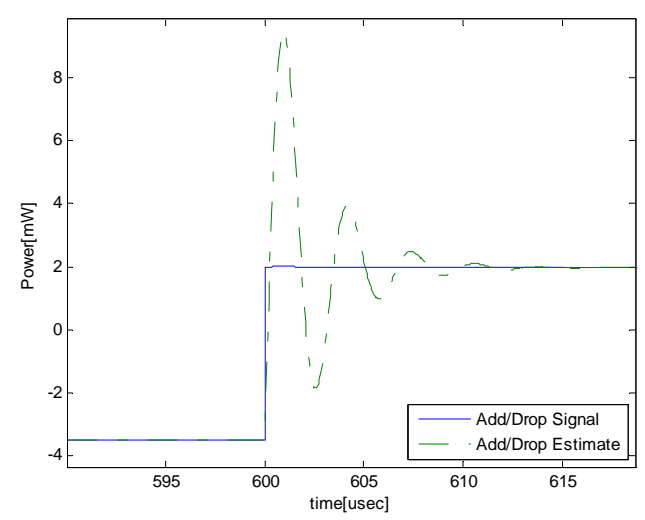

(b) Add/Drop Signal Estimation

Figure 5. Gain Response of Channel 1 in case of $3.5 \mathrm{~mW}$ channel 2 signal drop $\left(\omega_{n}=300000\right)$
Figure 6 summarizes the comparison between the responses when $\omega_{n}=300000 \quad(\mathrm{rad} / \mathrm{sec})$ and $\omega_{n}=100000 \quad(\mathrm{rad} / \mathrm{sec})$. Since the natural frequency $\omega_{n}=300000 \quad(\mathrm{rad} / \mathrm{sec})$ of the desired system is more than 3 times larger than the case of $\omega_{n}=100000(\mathrm{rad} / \mathrm{sec})$, the gain response is stabilized 3 times faster than the case of $\omega_{n}=100000 \quad(\mathrm{rad} / \mathrm{sec})$. On the other hand, the transient overshoots are almost the same for both cases since the DOB gain and damping ratio were chosen the same for both cases.

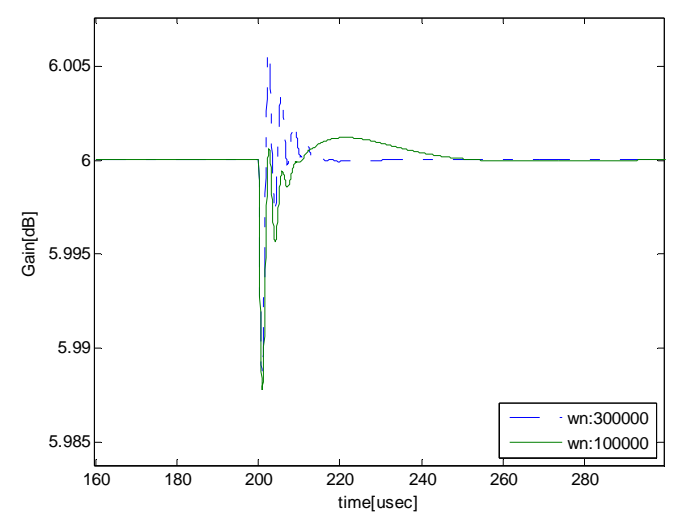

Figure 6. Comparison of Gain Responses between the cases of $\omega_{n}=100000$ and $\omega_{n}=300000$

\section{Conclusions}

In this paper, the performance analysis has been carried out for the proposed DOB based PI control algorithm for EDFA gain control. By introducing DOB to compensate gain fluctuations due to channel add/drops, the transient responses are stabilized much faster than PI controller without DOB. The proposed gain controller is composed of a disturbance observer and a PI controller. We have applied a disturbance observer technique to detect channel add/drop signals and theoretically proven the performance of the proposed method.

Since the channel add/drops are estimated by DOB and subtracted it feed forwardly, the gain responses are theoretically shown to rapidly converge to desired system responses after the transient responses decay to zero. Theoretical analysis has been done based on two-level EDFA model.

Simulation results showed that DOB can suppress large fluctuations in the beginning of 
channel add/drops and result in faster settling to desired channel gains.

As mentioned in Simulations section, we should consider the exact EDFA model in order to enhance the performance of DOB but the exact three-level model does not satisfy matching condition and then conventional DOB technique cannot be directly applied.

\section{Acknowledgements}

This research was supported by Hallym University Research Fund 2012 (HRF-201211014) and by Mid-career Researcher Program (No.2011-0013091, 2012-005324) through the National Research Foundation of Korea(NRF) funded by the Ministry of Education, Science, and Technology.

\section{REFERENCES}

1. PAVEL, L., Dynamics and Stability in Optical Communication Networks: A System Theoretic Framework, Automatica, vol. 40, no. 8, 2004, pp. 1361-1370.

2. ZYSKIND, J. L., et al., Fast Power Transients in Optically Amplified Multiwavelength Optical Networks, Proceedings of OFC'96, 1996, Paper PD31.

3. SRIVASTAVA, A. K., Y. SUN, J. L. ZYSKIND, J. W. SULHOFF, EDFA Transient Response to Channel Loss in WDM Transmission System, IEEE Photonics Technology Letters, vol. 9, no. 3, Mar. 1997, pp. 386-388.

4. SUN, Y., et al., Fast Power Transients in WDM Optical Networks with Cascaded EDFAs, Electronics Letters, vol. 33, no. 4, 1997, pp. 313-314.

5. ZIRNGIBL, M., Gain Control in Erbium-doped Fiber Amplifiers by Alloptical Feedback Loop, Electronics Letters, vol. 27, no.7, 1991 , pp. 560-561
6. DESURVIRE, E., Erbium Doped Fiber Amplifiers: Principles and Applications. New York, Wiley, 1994.

7. TRAN, A. V., C. J. CHAE, R. S. TUCKER, Y. J. WEN, EDFA Transient Control based on Envelope Detection for Optical Burst Switched Networks, IEEE Photonics Technology Letters, vol. 17, no. 1, January 2005, pp. 226-228.

8. SRIVASTAVA, A. K. et al., Fast-link Control Protection of Surviving Channels in Multi-wavelength Optical Networks, IEEE Photonics Technology Letters, vol. 9, no. 12, Dec. 1997, pp. 1667-1669.

9. TAING, Y., L. PAVEL, An EDFA H Controller for Suppression of Power Excursions Due to Pilot Tones and Network Traffic, IEEE Photonics Technology Letters, vol. 18, no. 18, September 15, 2006, pp. 1916-1918.

10. SHIN, S., D. KIM, S. KIM, S. LEE, S. SONG, A Novel Technique to Minimize Gain-transient Time of WDM Signals in EDFA, Journal of the Optical Society, Korea, vol. 10, no. 4, 2006, pp. 174-177.

11. KOKOTOVIC, K. K., H. KHALIL, J. ORELLY, Singular Perturbation Method in Control, SIAM, 1987.

12. CHOI, Y., W. K. CHUNG, Y. YOUM, Disturbance Observer in Framework, IEEE IECON, 1996, pp. 1394-1400.

13. KIM, K., H. T. CHOI, W. K. CHUNG, I. H. SUH, Analysis and Design of Robust Motion Controllers in the Unified Framework, Journal of Dynamic Systems, Measurement, and Control, vol. 124, 2002, pp. 313-321.

14. DESURVIRE, E., Erbium-doped Fiber Amplifiers, John Wiley \& Sons, New York, 1994.

15. KHALIL, H. K. Nonlinear Systems, 2nd Ed., Prentice-Hall, 2002 


\section{Appendix}

\section{Proof of Theorem 1}

We define Lyapunov function as follows.

$V=E^{T} P E$

where

$E=\left[\begin{array}{ll}E_{1} & E_{2}\end{array}\right]^{T}, E_{1}=\left[\begin{array}{ll}e_{1} & e_{2}\end{array}\right]^{T}, E_{2}=e_{3}$.

Then, we have the following equations from (34) and (35).

$$
\begin{aligned}
\frac{d}{d t} E_{1} & =A_{1} E_{1}-B E_{2} \\
\frac{d}{d t} E_{2} & =-\bar{A} E_{2}-K(t)\left\{\left[-K_{I}-\frac{\Gamma_{21}+B_{k} K_{p}}{B_{k}}\right] E_{1}-E_{2}\right\} \\
& -K(t)\left\{-\frac{\Gamma_{21}+B_{k} K_{p}}{B_{k}}\left(\bar{G}_{k}-G_{k}^{C}\right)-K_{I} \int_{0}^{t}\left(\bar{G}_{k}-G_{k}^{C}\right) d t\right\}
\end{aligned}
$$

The time derivative of Lyapunov function $\mathrm{V}$ is satisfying the following inequalities using (31), (32), (34), (36), (37) and (38).

$$
\begin{aligned}
\frac{d}{d t} V & \leq E_{1}^{T}\left(A_{1}^{T} P_{1}+P_{1} A_{1}\right) E_{1}-2 E_{1}^{T} P_{1} B E_{2} \\
& -2 \bar{A} E_{2}^{2}-2 K(t) E_{2}\left\{\left[-K_{I}-\frac{\Gamma_{21}+B_{k} K_{p}}{B_{k}}\right] E_{1}-E_{2}\right\} \\
& -2 K(t) E_{2}\left\{-\frac{\Gamma_{21}+B_{k} K_{p}}{B_{k}}\left(\bar{G}_{k}-G_{k}^{C}\right)-K_{I} \int_{0}^{t}\left(\bar{G}_{k}-G_{k}^{C}\right) d t\right\} \\
& \leq-E_{1}^{T} Q_{1} E_{1}-2\left\|E_{1}\right\||| P_{1} B \| E_{2} \mid \\
& -2(\bar{A}-\bar{K}) E_{2}^{2}+2 \bar{K} \sqrt{K_{I}^{2}+\left(\frac{\Gamma_{21}+B_{k} K_{p}}{B_{k}}\right)^{2}}\left|E_{2} \| E_{1}\right| \\
& +2 \bar{K})\left|E_{2}\right|\left\{\frac{\Gamma_{21}+B_{k} K_{p}}{B_{k}} \gamma_{G} \| \Theta\left(0\left\|e^{-\sigma t}+K_{I} \gamma_{I}\right\| \Theta\left(0 \| e^{-\sigma t}\right\}\right.\right. \\
& \left.\leq-E^{T} \bar{Q} E+2 \bar{K}\right)\left|E_{2}\right|\left\{\frac{\Gamma_{21}+B_{k} K_{p}}{B_{k}} \gamma_{G} \| \Theta\left(0\left\|e^{-\sigma t}+K_{I} \gamma_{I}\right\| \Theta\left(0 \| e^{-\sigma t}\right\}\right.\right. \\
& \leq-\frac{\lambda_{m}(\bar{Q})}{\lambda_{M}(P)} V \\
& +2 \sqrt{\frac{1}{\lambda_{m}(P)}} \bar{K}\left\{\frac{\Gamma_{21}+B_{k} K_{p}}{B_{k}} \gamma_{G} \| \Theta\left(0\left\|e^{-\sigma t}+K_{I} \gamma_{I}\right\| \Theta\left(0 \| e^{-\sigma t}\right\} V^{\frac{1}{2}}\right.\right.
\end{aligned}
$$

where 
$\bar{Q}=\left[\begin{array}{cc}\lambda_{m}(Q) & \left\|P_{1} B\right\|+\bar{K} \sqrt{K_{I}^{2}+\left(\frac{\Gamma_{21}}{B_{k}}+K_{p}\right)} \\ \left\|P_{1} B\right\|+\bar{K} \sqrt{K_{I}^{2}+\left(\frac{\Gamma_{21}}{B_{k}}+K_{p}\right)} & \bar{A}-\bar{K}\end{array}\right]$

(A.4) can be rewritten as follows.

$$
\begin{aligned}
\frac{d}{d t} V^{\frac{1}{2}} & \leq \frac{\lambda_{m}(\bar{Q})}{\lambda_{M}(P)} V^{\frac{1}{2}} \\
& +\sqrt{\frac{1}{\lambda_{m}(P)}} \bar{K}\left\{\frac{\Gamma_{21}+B_{k} K_{p}}{B_{k}} \gamma_{G} \mid \Theta\left(q\left|e^{-\alpha}+K_{I} \gamma_{I}\right| \operatorname{er}\left(q \mid e^{-\alpha}\right\}\right.\right.
\end{aligned}
$$

From (A.5) and comparison principle [15], we have the following.

$$
V^{\frac{1}{2}}(t) \leq V^{\frac{1}{2}}(0) e^{-\sigma_{V} t}+\tilde{K} \frac{1}{\left|\sigma_{V}-\sigma\right|} e^{-\sigma t}
$$

where

$$
\begin{aligned}
& \sigma_{V}=\frac{\lambda_{m}(\bar{Q})}{\lambda_{M}(P)}, \\
& \tilde{K}=\sqrt{\frac{1}{\lambda_{m}(P)}} \bar{K}\left\{\frac{\Gamma_{21}+B_{k} K_{p}}{B_{k}} \gamma_{G}+K_{I} \gamma_{I}\right\} \| \Theta(0 \|
\end{aligned}
$$

From (A.6) and (A.1), the error variables asymptotically converge to zero. 